Vassilopoulos et al reported three adult cases, where two were not treated with immunosuppressive drugs before anakinra and one had recurrence after anakinra was tapered. The Double Blind Placebo Controlled Clinical Trial AIRTRIP, shows efficacy of anakinra in treating 11 patients with recurrent pericarditis over 14 months. It is unclear from this study if anakinra should be tapered or not. We suggest that once steroids and immunosuppressive drugs have been discontinued, anakinra should be gradually tapered over months, to avoid relapse. These experiences warrant further long term controlled trials in order to determine the efficacy and appropriate treatment regimen of anakinra for recurrent pericarditis.

Disclosure of Interest: None declared

DOI: 10.1136/annrheumdis-2017-eular.1141

\section{THU0571 THE CLINICAL FEATURES OF 223 BEHCET'S DISEASE PATIENTS IN JAPAN}

R. Saito ${ }^{1}$, K. Nishimura ${ }^{1}$, H. Mukoyama ${ }^{1}$, Y. Nakamura ${ }^{1}$, T. Nagamoto ${ }^{1}$, K. Akashi ${ }^{2}$, A. Onishi ${ }^{2}$, Y. Kogata ${ }^{2}$, J. Saegusa ${ }^{2}$, A. Morinobu ${ }^{2}$, T. Yokota ${ }^{1}$. ${ }^{1}$ Department of Endocrinology and Rheumatology, Kurashiki Central Hospital, Kurashiki; ${ }^{2}$ Department of Rheumatology and Clinical Immunology, Kobe University Graduate School of Medicine, Kobe, Japan

Background: Behcet's disease is a systemic vasculitis disease with oral and genital aphthous ulcers, ocular involvements, skin manifestations, arthritis, gastrointestinal manifestations, neurogenic diseases and vascular involvements. Patients with Behcet's disease are known to distribute along the ancient Silk Road, including Japan.

Objectives: We evaluate the clinical features of Behcet's disease in Japan. Methods: We retrospectively investigated 223 patients (108 males and 115 females) who fulfilled the International Criteria for Behcet's Disease (ICBD) from January, 2006 until May, 2015. We examined sex, onset age, disease type, clinical symptoms, laboratory data and medications.

\section{Results:}

Median age at diagnosis was $36.0 \pm 12.8$ years old. Oral ulcers were the most common manifestation $(98.2 \%)$, followed by genital ulcers $(62.4 \%)$, ocular involvements $(53.2 \%)$, erythema nodosum $(53.2 \%)$, acneiform lesions $(51.8 \%)$, arthritis $(38.6 \%)$, gastrointestinal manifestations $(25.1 \%)$, neurogenic diseases $(9.0 \%)$, and vascular involvements $(8.1 \%)$. The relationship of HLA and disease manifestations was studied in 123 patients (41.5\% with HLA-B51 and $24.1 \%$ with HLA-A26). The frequency of acneiform lesions, ocular involvements and HLA-B51 was significantly higher in male, while genital ulcers and arthritis were significantly higher in female. Patients with ocular involvements showed a higher assosiation rate with neurogenic diseases and HLA-B51, and lower wih gastrointestinal manifestations. TNF $\alpha$ inhibitor (infliximab or adalimumab) were used for 66 cases $(30 \%)$, and it could be continued for 1 year in $91 \%$, and for 2 years in $83 \%$.

\begin{tabular}{|c|c|c|c|c|}
\hline & Total $(\mathrm{n}=223)$ & Male $(n=108)$ & Female $(n=115)$ & $\mathrm{pva}$ \\
\hline Oral ulcers & $218 / 222(98.2 \%)$ & $105 / 107(98.1 \%)$ & $113 / 115(98.3 \%)$ & 0.94 \\
\hline Skin manifestations & $190 / 220(86.4 \%)$ & $90 / 107(84.1 \%)$ & $100 / 113(88.5 \%)$ & 0.34 \\
\hline Erythema nodosum & $116 / 218(53.2 \%)$ & $49 / 105(46.7 \%)$ & $67 / 113(59.3 \%)$ & 0.06 \\
\hline Acneifor & $113 / 21$ & $65 / 105$ & $48 / 113$ & 0.004 \\
\hline Thrombophlebit & $11 / 218(5.0 \%)$ & $5 / 105(4.8 \%)$ & $6 / 113(5.3 \%)$ & 0.85 \\
\hline ments & $118 / 222(53.2 \%)$ & $68 / 108$ & $50 / 114$ & 0.004 \\
\hline Genital ulcers & $138 / 221(62.4 \%)$ & $58 / 107(54.2 \%)$ & $80 / 114(70.2 \%)$ & 0.01 \\
\hline Artiritis & $86 / 223(38.6 \%)$ & $33 / 108$ (30.6\%) & $53 / 115(46.1 \%)$ & 0.02 \\
\hline Gastroin & $56 / 223(2$ & $23 / 108$ & $33 / 115(28.7 \%)$ & 0.20 \\
\hline Neurc & $20 / 223$ & $12 / 10$ & $8 / 115$ & 0.28 \\
\hline Vascula & $18 / 223(8.1 \%)$ & $6 / 108(5.6 \%)$ & $12 / 115(10.4 \%)$ & 0.18 \\
\hline HLA-B! & $51 / 123(41.5 \%)$ & $32 / 62(51.6 \%)$ & $19 / 61(31.1 \%)$ & 0.02 \\
\hline HLA- & $28 / 116(24.1 \%)$ & $14 / 56(25.0 \%)$ & $14 / 60(23.3 \%)$ & 0. \\
\hline
\end{tabular}

\begin{tabular}{lcccc} 
Table 2 & \multicolumn{5}{l}{} & \\
\hline & $\begin{array}{c}\text { Total } \\
(\mathrm{n}=223)\end{array}$ & $\begin{array}{c}\text { With ocular } \\
\text { involvements }(\mathrm{n}=118)\end{array}$ & $\begin{array}{c}\text { Without ocular } \\
\text { involvements }(\mathrm{n}=104)\end{array}$ & $\mathrm{p}$ value \\
\hline Gastrointestinal & & & & \\
$\quad$ manifestations & $56 / 223(25.1 \%)$ & $17 / 118(14.4 \%)$ & $39 / 104(37.5 \%)$ & $<0.001$ \\
Neurogenic diseases & $20 / 223(9.0 \%)$ & $19 / 118(16.1 \%)$ & $1 / 104(1.0 \%)$ & $<0.001$ \\
Vascular involvements & $18 / 223(8.1 \%)$ & $7 / 118(5.9 \%)$ & $11 / 104(10 \% \%)$ & 0.21 \\
HLA-B51 positive & $51 / 123(41.5 \%)$ & $36 / 69(52.2 \%)$ & $15 / 54(27.8 \%)$ & 0.006 \\
HLA-A26 positive & $28 / 116(24.1 \%)$ & $19 / 65(29.2 \%)$ & $9 / 51(17.6 \%)$ & 0.15 \\
\hline
\end{tabular}

Conclusions: A higher incidence of gastrointestinal manifestations was observed in patients with Behcet's disease in Japan. Patients with ocular involvements showed a higher assosiation rate with neurogenic diseases, and lower wih gastrointestinal manifestation. Most patients could continue TNF $\alpha$ inhibitor safety and effectively.

Disclosure of Interest: None declared

DOI: 10.1136/annrheumdis-2017-eular.4216

\section{THU0572 ASSOCIATION BETWEEN RETROPERITONEAL FIBROSIS AND MALIGNANCY: A POSSIBLE PARANEOPLASTIC SYNDROME}

S.J. Lee ${ }^{1}$, J.S. Eun ${ }^{1}$, E.Y. Lee ${ }^{2}$, G.B. Bae ${ }^{1}$, E.J. Nam ${ }^{1}$, Y.W. Song ${ }^{2}$, Y.M. Kang ${ }^{1} .{ }^{1}$ Internal Medicine, Division of Rheumatology, Kyungpook National University Hospital, Daegu; ${ }^{2}$ Internal Medicine, Division of Rheumatology, Seoul National University Hospital, Seoul, Korea, Republic Of

Background: Retroperitoneal fibrosis (RPF) are associated with malignancies. However it is unclear what is the incidence of malignancies and whether particular malignancies are more prevalent in RPF.

Objectives: The objective of this study was to examine standardized incidence ratios (SIRs) of cancers in patients with retroperitoneal fibrosis (RPF) compared with age- and sex-matched general population.

Methods: Medical records of 111 patients diagnosed as having RPF by computed tomography, positron emission tomography and/or histological evaluation were reviewed. Forty one cases of cancers, which were confirmed by biopsies, were identified in 35 patients with RPF. SIRs were calculated for cancers, cancer types, and age at cancer diagnosis and stratified according to RPF-cancer intervals compared with general population in Korea.

Results: The mean \pm SD age at RPF diagnosis was $59.1 \pm 14.9$ years, and $69.4 \%$ of the patients were male. The cancer SIR (95\% confidence intervals) in patients with RPF relative to age- and sex-matched individuals in the general population was $3.18(2.23$ - 4.41) [2.65 (1.7 - 3.94) in men; 5.34 (2.76 - 9.32) in women]. The most frequent cancer was unspecified urinary organ cancers with SIR of 733.41 (238.14 - 1711.53). SIRs of multiple myeloma [27.58 (3.34 - 99.64)], renal cell cancers [9.53, (1.15 - 34.42)] and unspecified cancers [16.92, (2.05 - 61.12)] were also significantly higher than in general population. Whereas cancers were most frequently developed in the eighth decade of life, the peak SIR was observed in the fifth decade $(8.41,2.29-21.53)$. When stratified by RPF-cancer intervals, SIR was 6.85 (4.55 - 9.90) within 2 years of RPF diagnosis, while no significant increase in SIR was found out of 2 years. Malignancies $(n=28)$ within 2 year of RPF diagnosis included unspecified urinary organ cancer $(n=4)$, stomach cancer $(n=4)$, lung cancer $(n=4)$, colon cancer $(n=3)$, renal cell cancer $(n=2)$, pancreatic cancer $(n=2)$, unspecified cancer $(n=2)$, rectal cancer $(n=1)$, gallbladder cancer $(n=1)$, non-Hodgkin lymphoma $(n=1)$, multiple myeloma $(n=1)$, prostate cancer $(n=1)$, thyroid cancer $(n=1)$ and gastrointestinal stromal tumor $(n=1)$. Predominant origin of these malignancies were epithelial cell types [transitional cell carcinomas $(n=4)$, adenocarcinoma $(n=16)]$.

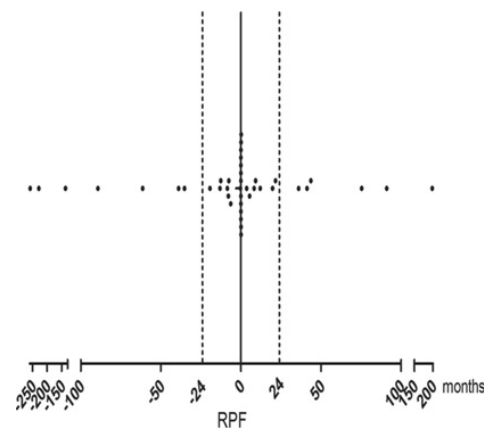

Conclusions: RPF was strongly associated with cancers, particularly within 2 years of RPF diagnosis. Our results indicate that cancer screening in patients with RPF should may be performed regularly up to 2 years after RPF diagnosis. References:

[1] Temporal relationship between cancer and myositis identifies two distinctive subgroups of cancers: impact on cancer risk and survival in patients with myositis. Kang EH, Lee SJ et al Rheumatology (Oxford). 2016 Sep;55(9):163141.

Disclosure of Interest: None declared

DOI: 10.1136/annrheumdis-2017-eular.6168

\section{THU0573 CONCOMITANT AUTOIMMUNE DISEASES IN PATIENTS WITH SARCOIDOSIS}

S. Kobak ${ }^{1}$, F. Sever ${ }^{2}$, H. Semiz ${ }^{3}$, M. Orman ${ }^{4} .{ }^{1}$ Rheumatology, Istinye University Faculty of Medicine, LIV Hospital, Istanbul; ${ }^{2}$ Chest Diseases, Medicalpark Hospital; ${ }^{3}$ Internal Medicine; ${ }^{4}$ Statistics, Ege University Faculty of Medicine, Izmir, Turkey

Background: Sarcoidosis is a chronic granulomatous disease characterized by non-caseating granuloma formation. It can mimic many rheumatic diseases and/or may be coexist with them. There are limited data in the literature about the association of sarcoidosis with autoimmune diseases.

Objectives: The purpose of this study is to determine the frequency and characteristics of autoimmune diseases associated with patients with sarcoidosis. Methods: One hundred and thirty-one sarcoidosis patients folowed-up in single rheumatology center were included in the study. Demographic, clinical, laboratory and radiological data of these cases were evaluated retrospectively. The 